\title{
Expert consensus report on remote monitoring of implantable devices: the Dutch experience
}

\author{
C. C. de Cock • J. Elders • N. M. van Hemel
}

Published online: 13 January 2012

(C) The Author(s) 2012. This article is published with open access at Springerlink.com

The annual absolute number of cardiovascular implantable devices (CIEDs) is steadily increasing and is expected to rise even further, mostly due to the ageing demographic changes. [1] Consequently this will have a major impact on the postimplantation follow-up of monitor device function and patient condition both in terms of time and personnel. In a recent survey in 14 European countries remote monitoring (RM) was used in about $85 \%$ of all centres and the responding centres estimated that this percentage will increase to $93 \%$ by 2015 . [2] In the same survey, guidelines and/or recommendations for the use of RM available from national scientific societies were reported to be totally absent in $93 \%$. This lack of structural guidelines and/or recommendations will undoubtedly lead to a wide heterogeneity among centres using RM for the optimal care of patients after implantation. In addition responsibility related to remote follow-up is not clearly defined and leaves the device specialist and allied professionals in doubt with respect to the legal issues of RM. In 2009 the Netherlands Heart Rhythm Association (NHRA) took the initiative to develop a consensus document for RM in order to make a more uniform approach for RM and remote follow-up of the device patient.

\footnotetext{
C. C. de Cock $(\bowtie)$

Department of Cardiology, VU University Medical Center, Boelelaan 1117,

$1081 \mathrm{HV}$, Amsterdam, the Netherlands

e-mail: cc.dcock@vumc.nl

J. Elders

Department of Cardiology, Canisius Wilhelmina Hospital,

Weg door Jonkerbos 100 ,

6500 GS, Nijmegen, the Netherlands

N. M. van Hemel

Heart Lung Center, Utrecht University,

P.O. Box 80125, Utrecht, the Netherlands
}

Close to 60 individuals have contributed to this expert consensus report. All aspects related to RM were divided into subgroups focussing on well-defined issues. The following subgroups were defined:

1. Description of remote CIED monitoring

2. Indications, benefits and limitations of remote CIED monitoring

3. Patient perception of remote CIED monitoring

4. Contribution of manufacturer to remote CIED monitoring

5. Costs and reimbursement of remote CIED monitoring

6. Preliminary recommendations for remote CIED monitoring

For each subgroup a working group was formed. For each working group a chairperson was appointed and 5 to 8 members were carefully selected based on personal experience and individual expertise in the field. During half-day sessions working groups focussed on their specific subject which resulted in a concept document which was subsequently distributed by the chairperson for additional review.

All sessions were also attended by the first three authors of the final expert consensus documents ( $\mathrm{CdC}, \mathrm{JE}$ and $\mathrm{NvH})$ for process surveillance without interference with the contents. Finally the concept consensus report was reviewed during half-day sessions with all chairpersons.

Despite the fact that RM was introduced more than 10 years ago, guidelines are available in only $5 \%$ of European countries, although RM is already widely implemented in medical practice. [2]

A uniform approach to patients with CIED permitting RM is essential for a safe and optimal treatment. Clear and standardised information to the patient is also needed for optimal transmissions. 
In the CONNECT trial up to $45 \%$ of all alerts were not transmitted simply because the setup of the system was inappropriate.[3] In the Dutch expert consensus document a brochure is presented with patient instructions and education emphasising the responsibilities of the patient with respect to RM. [4]

In addition a minimal set of parameters and alerts has been defined which may impose early medical treatment and prevent appropriate and inappropriate shocks. [5] Furthermore the introduction of RM into a device clinic needs a tailored organisation with structured appointments on scheduled or unscheduled visits, reason and type of device reprogramming etc. Of interest, the present expert consensus document has made a clear statement that RM can and will not be capable to be used as a 24/7 emergency system.

The Heart Rhythm Society/European Heart Rhythm Association expert consensus document on monitoring of CIEDs was published in 2008 and lacks a clear statement on $24 / 7$ surveillance, which fuelled concern with respect to legal issues for alerts.

Finally, reimbursement will be a key issue for a wide implementation of RM. For private insurance companies and national health services the major challenge is in demonstrating improved health care with no rise in health rise costs. [6] In $82 \%$ of the countries in Europe there is no reimbursement structure despite the fact that preliminary studies demonstrated the cost effectiveness of RM and a high patient acceptance and satisfaction. [7] More prospective studies are needed with health economic costs as one of the primary endpoints before RM will be standard of care for all patients after device implantation. [8,9]
Open Access This article is distributed under the terms of the Creative Commons Attribution Noncommercial License which permits any noncommercial use, distribution, and reproduction in any medium, provided the original author(s) and source are credited.

\section{References}

1. EUCOMED (European Medical Technology Industry Association) http://www.eucomed.com

2. Halimi F, Cantú F, on behalf of the European Heart Rhythm Association (EHRA) Scientific Initiatives Committee (SIC). Remote monitoring for active cardiovascular electronic devices: a European survey. Europace. 2010;12:1778-80.

3. Crossley G, Boyle A, Vitense H, et al. for the CONNECT investigators. The CONNECT (Clinical Evaluation of Remote Notification to Reduce Time to Clinical Decision) Trial. J Am Coll Cardiol. 2011;57:1181-9.

4. Remote monitoring and follow-up of cardiovascular implantable electronic devices in the Netherlands. An expert consensus report of the Netherlands Society of Cardiology. http://www.nvvc.nl/home

5. Ricci RP, Morichelli L, Santini M. Home monitoring remote control of pacemaker and ICD patients in clinical practice. Impact on medical management and health care recource utilization. Europace. 2008;10:164-70.

6. Raatikainen MJ, Uusimaa P, van Ginneken MM, et al. Remote monitoring of implantable cardioverter defibrillator patients; a safe, time-saving and cost-effective means for follow-up. Europace. 2008;10:1145-51.

7. Ricci RP, Morichelli L, Quarta L, et al. Long-term patient acceptance of and satisfaction with implanted device remote monitoring. Europace. 2010;12:674-9.

8. Cock CC, Elders J, van Hemel NM, et al. Remote monitoring and follow-up of cardiovascular implantable electronic devices in the Netherlands. An expert consensus report of the Netherlands Society of Cardiology. Neth Heart J 2012;20:xx-xx

9. van der Wall EE. Remote monitoring: coming close? Neth Heart J. 2010;18:283. 